\title{
OSCILLATION THEOREMS FOR SECOND-ORDER DIFFERENTIAL EQUATIONS WITH FUNCTIONAL ARGUMENTS
}

\author{
CURTIS C. TRAVIS
}

ABstract. The oscillatory behavior of $Y^{\prime \prime}(t)+P(t) Y(g(t))=0$ where $g(t) \rightarrow \infty$ as $t \rightarrow \infty$ is investigated. Sufficient conditions for the oscillation of $Y^{\prime}(t)$ and $Y(t)$ are developed.

1. Introduction. The purpose of this paper is to study the oscillatory behavior of the differential equation

$$
Y^{\prime \prime}(t)+P(t) Y(g(t))=0 .
$$

It is assumed throughout that $P(t)$ and $g(t)$ are continuous on $[a, \infty)$ and that $g(t) \rightarrow \infty$ as $t \rightarrow \infty$. A nontrivial solution to (1.1) is called oscillatory if it exists on a half-line and has arbitrarily large zeros. A solution is called nonoscillatory if it is eventually of one sign. Equation (1.1) is called oscillatory if every solution is oscillatory.

2. Main results. Waltman [5] and Bradley [2] have recently demonstrated that all solutions of (1.1) are oscillatory if $P(t) \geqq 0$ and $\int^{\infty} P(t) d t=$ $\infty$.

If $g(t) \equiv t$, equation (1.1) reduces to the ordinary differential equation

$$
Y^{\prime \prime}(t)+P(t) Y(t)=0 \text {. }
$$

It is a well-known theorem of Wintner [6] and Leighton [3] that $\int^{\infty} P(t) d t=\infty$ is sufficient for equation (2.1) to be oscillatory even when $P(t)$ is not assumed positive. It is also known [1] that all solutions of the nonlinear equation

$$
\begin{gathered}
Y^{\prime \prime}(t)+P(t) h(Y(t))=0, \\
h(x) x>0 \quad \text { for } x \neq 0, \\
h^{\prime}(x) \geqq 0 \quad \text { for all } x,
\end{gathered}
$$

oscillates if $\int^{\infty} P(t)=\infty$, where $P(t)$ is not assumed positive. On the basis

Received by the editors February 16, 1971 and, in revised form, April 19, 1971 and May 3, 1971.

AMS 1970 subject classifications. Primary 34K15.

Key words and phrases. Oscillation, functional arguments, delay equation, strongly oscillatory coefficient.

(c) American Mathematical Society 1972 
of these two results, one might conjecture that the Leighton-Wintner oscillation theorem could be extended to equations of the form (1.1). The equation $Y^{\prime \prime}(t)+((\sin t) /(2-\sin t)) Y(t-\pi)=0$ demonstrates that this is not the case. This equation has the nonoscillatory solution $Y(t)=$ $2+\sin t$ even though $\int^{\infty}((\sin t) /(2-\sin t)) d t=\infty$. However this solution does have the property that $Y^{\prime}(t)$ has arbitrarily large zeros. Theorem 2.1 below shows that the derivative of any solution of an equation of form (1.1) has arbitrarily large zeros if we assume $g^{\prime}(t) \geqq 0$ and $\int^{\infty} P(t) d t=\infty$.

THEOREM 2.1. Assume $g(t)$ is differentiable, $g^{\prime}(t) \geqq 0$, and $\int^{\infty} P(t) d t=$ $\infty$. Then $Y^{\prime}(t)$ has arbitrarily large zeros for any solution $Y(t)$ of $(1.1)$.

PRoof. If $Y(t)$ oscillates then the conclusion of the theorem is certainly true. If $Y(t)$ is ultimately positive, then so is $Y(g(t))$. Suppose $Y^{\prime}(t)>0$ for all large $t$. Then $W(t)=Y^{\prime}(t) / Y(g(t))$ satisfies the equation

Integrating we obtain

$$
W^{\prime}(t)=-P(t)-W(t) \frac{Y^{\prime}(g(t))}{Y(g(t))} g^{\prime}(t) \leqq-P(t) .
$$

$$
W(x) \leqq W(\alpha)-\int_{\alpha}^{x} P(t) d t .
$$

Since $\int^{\infty} P(t) d t=\infty$ we have a contradiction. Suppose now that $Y^{\prime}(t)<0$ for all large $t$. It is not difficult to see that $\int^{\infty} P(t) d t=\infty$ implies that there exists $T$ sufficiently large so that

Hence, we have

$$
\int_{T}^{t} P(s) d s \geqq 0 \quad \text { for } t \geqq T .
$$

$$
\begin{aligned}
& \int_{T}^{t} P(s) Y(g(s)) d s \\
& \quad=Y(g(t)) \int_{T}^{t} P(s) d s-\int_{T}^{t} Y^{\prime}(g(s)) g^{\prime}(s) \int_{T}^{s} P(r) d r d s \geqq 0, \quad t \geqq T .
\end{aligned}
$$

Now integrating equation (1.1) we have $Y^{\prime}(t) \leqq Y^{\prime}(T)<0$ which contradicts the fact that $Y(t)$ is positive for large $t$. This completes the proof.

Considering the equation

$$
Y^{\prime \prime}(t)+\lambda P(t) Y(t)=0,
$$

we shall call $P(t)$ a strongly oscillatory coefficient if (2.2) is oscillatory for all positive $\lambda$. If $P(t) \geqq 0$, Nehari [4] shows that

$$
\limsup _{x \rightarrow \infty} x \int_{x}^{\infty} P(t) d t=\infty
$$


is a necessary and sufficient condition for $P(t)$ to be a strongly oscillatory coefficient.

In Theorem 2.2 below, we shall consider the equation

$$
Y^{\prime \prime}(t)+P(t) f(Y(t), Y(g(t)))=0,
$$

where $P, g$, and $f$ are assumed continuous, and $f(y, W)$ has the sign of $y$ and $W$ when they have the same sign. We will show that if $P(t)$ is a positive strongly oscillatory coefficient and if $g(t)$ increases "sufficiently fast," then any solution of $(2.3)$ that exists on a ray $(a, \infty)$ is oscillatory. In order to avoid the assumption that $g(t)$ is differentiable, we introduce a differentiable minorant $h(t)$. This will allow the theorem to be applied to delay equations of the form $Y^{\prime \prime}(t)+P(t) Y(t-\tau(t))=0,0 \leqq \tau(t) \leqq$ $M$, where $\tau(t)$ is not assumed differentiable.

THEOREM 2.2. If

(i) $h(t) \leqq g(t)$ and $0<k \leqq h^{\prime}(t) \leqq 1$,

(ii) there exists $M>0$ such that $y \geqq M$ implies

$$
\liminf _{|w| \rightarrow \infty}\left|\frac{f(y, w)}{w}\right| \geqq \epsilon>0,
$$

(iii) $P(t) \geqq 0$ and $\lim \sup _{x \rightarrow \infty} x \int_{x}^{\infty} P(t) d t=\infty$, then all solutions of $(2.3)$ existing on $(a, \infty)$ are oscillatory.

Proof. Assume the contrary. Then (2.3) has a nonoscillatory solution $Y(t)$. Assume that $Y(t)>0$ for large $t$ (the case $Y(t)<0$ can be treated similarly). It is easy to verify that $Y^{\prime}(t)>0$ for large $t$. Let $W(t)=$ $Y^{\prime}(t) / Y(h(t))$. Then $W(t)$ satisfies

$$
W^{\prime}(t)=-P(t) \frac{f(Y(t), Y(g(t)))}{Y(h(t))}-\frac{Y^{\prime}(h(t))}{Y(h(t))} h^{\prime}(t) W(t) .
$$

Since $Y^{\prime}(t)>0$ for large $t, \lim _{t \rightarrow \infty} Y(t)$ exists either as a finite or infinite limit. If $\lim _{t \rightarrow \infty} Y(t)=b$ is finite, then

$$
\lim _{t \rightarrow \infty} \frac{f(Y(t), Y(g(t)))}{Y(g(t))}=\frac{f(b, b)}{b}>0 .
$$

If $\lim _{t \rightarrow \infty} Y(t)=\infty$, then by (ii) we have that

$$
\frac{f(Y(t), Y(g(t)))}{Y(g(t))} \geqq \epsilon>0
$$

for large $t$. In either case, we have that (2.4) holds for sufficiently large $t$. 
Now since $Y(t)$ is increasing for large $t$ we have that

and also that

$$
P(t) \frac{f(Y(t), Y(g(t)))}{Y(h(t))} \geqq P(t) \frac{f(Y(t), Y(g(t)))}{Y(g(t))} \geqq \epsilon P(t)
$$

$$
\frac{Y^{\prime}(h(t))}{Y(h(t))} h^{\prime}(t) W(t) \geqq k W^{2}(t) .
$$

Therefore $W^{\prime}(t) \leqq-\epsilon P(t)-k W^{2}(t)$ and if we define $R(t)=k W(t)$, we obtain $R^{\prime}(t)+R^{2}(t)+k \epsilon P(t) \leqq 0$. However, by a well-known theorem of Wintner [7] this implies that the equation $Y^{\prime \prime}(t)+k \epsilon P(t) Y(t)=0$ is nonoscillatory. But this contradicts the fact that (iii) implies $P(t)$ is a strongly oscillatory coefficient.

In Theorem 2.2 we made an assumption on the rate of increase of $g(t)$. The equation

$$
Y^{\prime \prime}(t)+\frac{1}{4}\left(t^{3} \ln t\right)^{-1 / 2} Y(\ln t)=0 \text { for } 1<t
$$

demonstrates that the assumption is necessary. This equation has the nonoscillatory solution $Y(t)=t^{1 / 2}$ even though $P(t)=\frac{1}{4}\left(t^{3} \ln t\right)^{-1 / 2}$ is a strongly oscillatory coefficient.

\section{REFERENCES}

1. N. P. Bhatia, Some oscillation theorems for second order differential equations, J. Math. Anal. Appl. 15 (1966), 442-446. MR 34 \#3017.

2. J. Bradley, Oscillation theorems for a second-order delay equation, J. Differential Equations 8 (1970), 397-403.

3. W. Leighton, The detection of the oscillation of solutions of a second order linear differential equation, Duke Math. J. 17 (1950), 57-61. MR 11, $248 ; 871$.

4. Z. Nehari, Oscillation criteria for second-order linear differential equations, Trans. Amer. Math. Soc. 85 (1957), 428-445. MR 19, 415.

5. P. Waltman, $A$ note on an oscillation criterion for an equation with a functional argument, Canad. Math. Bull. 11 (1968), 593-595. MR 38 \#6193.

6. A. Wintner, A criterion of oscillatory stability, Quart. Appl. Math. 7 (1949), 115-117. MR 10, 456.

7. - , On the non-existence of conjugate points, Amer. J. Math. 73 (1951), 368-380. MR 13, 37.

Department of Mathematics, University of California, Davis, California 95616

Current address: Department of Mathematics, Vanderbilt University, Nashville, Tennessee 37203 